\title{
Parámetros biológicos básicos para la conservación de Pseudocurimata lineopunctata (Characiformes, Curimatidae) en el Río Anchicayá, Valle del Cauca, Colombia
}

\author{
Sandra Lamouroux-López ${ }^{1}$, Merling López ${ }^{2}$ \& Héctor Flores ${ }^{3}$ \\ 1. Programa de Tecnología en Acuicultura, Universidad del Pacífico, Km 3 vía al aeropuerto, Buenaventura, Colombia; \\ sllamouroux@yahoo.es \\ 2. Tecnóloga en Acuicultura; tatilo_ta12@hotmail.com \\ 3. Departamento de Acuicultura, Facultad de Ciencias del Mar, Universidad Católica del Norte, Larrondo 1281, \\ Coquimbo, Chile; hflores@ucn.cl
}

Recibido 13-I-2016. Corregido 03-X-2016. Aceptado 03-XI-2016.

\begin{abstract}
Basic biological parameters for Pseudocurimata lineopunctata (Characiformes, Curimatidae) conservation in the Anchicayá River of the Cauca Valley, Colombia. Pseudocurimata lineopunctata represents an important nutritional source for local human communities in Colombia and Ecuador. Although the yearly catch of this fish is low $(590 \mathrm{~kg} /$ year), there are no restrictions on minimum size, and this species is categorized as vulnerable with moderate extinction risk by the Regional Autonomous Corporation of the Cauca Valley (Colombia). To support conservation programs, biological data of the target species are required. Therefore, the aims of this study were to describe the sex ratio, distribution, size to weight ratio, macroscopic stages of reproductive maturity, fecundity, and size at first sexual maturity for $P$. lineopunctata. For this, fish samples were captured with nets over nine months (February-October) in 2007. The total length $\left(\mathrm{L}_{\mathrm{t}}\right)$ of the captured fish ranged between 10.7 and $16.5 \mathrm{~cm}$, with total weight $\left(\mathrm{P}_{t}\right)$ between 25.0 and $67.5 \mathrm{~g}$. Females represented $52.6 \%$ of the sample group while males represented $47.4 \%$, and differences in sex ratio were observed in relation to fish size. For all sampled months, the fish captured showed an advanced maturity state. Based on analyses of the gonadosomatic index (IGS), gonadic index (IG), and Fulton's condition factor (K), and two spawning peaks were determined, one in June-July, and the other in September-October. The size at first sexual maturity was $9.2 \mathrm{~cm}$ for females and $10.1 \mathrm{~cm}$ for males. Absolute fecundity was estimated as 3598 oocytes/ $\mathrm{g}$. It is necessary to carry out additional investigations on the reproduction of this species, to support future repopulation programs. Rev. Biol. Trop. 65 (1): 239-253. Epub 2017 March 01.
\end{abstract}

Key words: sex ratio, size-weight ratio, reproduction, spawning, size at first sexual maturity, fecundity.

En Colombia se han registrado cerca de 1435 especies de peces limnéticos (Maldonado-Ocampo, Vari, \& Usma, 2008), siendo el segundo país a nivel mundial en diversidad de peces dulceacuícolas (Instituto de Investigación de Recursos Biológicos Alexander von Humboldt, 2012). La diversidad por zona hidrogeográfica es de 675 especies para el Amazonas, 109 en el Caribe, 197 para Magdalena-Cauca, 619 para el Orinoco y 164 para el Pacífico (Chaves \& Santamaría, 2006). De estas especies, 173 son importantes para el consumo humano (Lasso et al., 2011).
Parte importante de estas especies, se encuentra con algún grado de amenaza, donde su mayor problema es la pérdida de hábitats naturales, que se relaciona generalmente con la expansión de la frontera agrícola y ganadera (Valderrama et al., 2014). Un total de 53 especies de peces dulceacuícolas, se encuentran bajo algún grado de amenaza, donde el $60 \%$ se registra en la cuenca del río Magdalena, $24.5 \%$ en la cuenca del Amazonas y cerca del $23 \%$ en la cuenca del Orinoco (Mojica, Usma, ÁlvarezLeón, \& Lasso, 2012). Las variables que explican la presencia de especies amenazadas son la 
contaminación y la sobreexplotación, con una incidencia del $58.5 \%$ y $51.0 \%$ respectivamente (Valderrama et al., 2014).

La conservación de los recursos ícticos es un deber de toda la ciudadanía; sin embargo, hay una serie de recursos, que son extraídos, tanto para consumo humano como para acuariofilia, de los cuales no se tienen mayores antecedentes biológicos. Uno de estos recursos ícticos es Pseudocurimata lineopunctata (Boulenger, 1911), conocida en las comunidades como: nayo de pozo, comebarro, jojorro, sardina, guagabina. Esta especie se distribuye en Colombia en los Ríos Atrato y San Juan, en el departamento del Chocó y ríos Dagua, Anchicaya y afluentes del este en el Valle del Cauca; y en el Ecuador en ríos del nordeste de Esmeraldas (Grueso \& Lamouroux, 2008; Froese \& Pauly, 2015). Habita aguas con poca o ninguna corriente, mucha veces presente en pozas; pero también en aguas con mucho sedimento (Ministerio Del Ambiente, 2011); este estudio se realizó en la cuenca hidrográfica del Río Anchicayá, que está localizada en la vertiente del Pacífico de la cordillera Occidental, en la jurisdicción de los municipios de Buenaventura y Dagua, la cual se extiende desde el nacimiento de su río en el Parque Nacional Natural Farallones de Cali, hasta la bahía de Buenaventura (Corporación Autónoma Regional del Valle del Cauca (C.V.C.) y la Universidad del Valle, 1998). La pluviosidad es constante (precipitaciones hasta $183 \mathrm{~mm}$ en 24 horas, en mayor proporción nocturnas), una temperatura ambiente de $26^{\circ} \mathrm{C}$, y una humedad relativa del $86 \%$, se encuentra recubierta por extensa zona selvática conocida como bosque muy húmedo tropical (Tovar, 2004).

Esta especie es capturada para consumo humano local (Angulo \& García, 2010; Sánchez-Garcés \& Ortega-Lara, 2011), es un importante componente nutricional, que contribuye a la seguridad alimentaria de los habitantes de las comunidades locales, con capturas diarias y en épocas de abundancia, especialmente en el verano (Programa Colombia Forestal [PCF], 2006). Su disponibilidad para las comunidades étnicas es escasa (CAMAWA, 2000), producto de su baja abundancia (2.8\%) en el ensamble de peces (Angulo \& García, 2010).

Al ser un recurso capturado localmente, no posee criterios de captura de tallas mínimas (Autoridad Nacional de Acuicultura y Pesca [AUNAP], 2013). Su captura es baja y representa los 590 kg/año (Autoridad Nacional de Acuicultura y Pesca [AUNAP], 2014). Sin embargo, este recurso fue declarado vulnerable y con riesgo moderado de extinción por la Corporación Autónoma Regional del Valle del Cauca CVC (Castillo \& González 2007; Maldonado-Ocampo et al., 2012) y en la Reserva Ecológica Cotacachi-Cayapas (Critical Ecosystem Partnership Fund [CEPF], 2007), debido a que su distribución se encuentra restringida a un área y con relativamente pocas poblaciones (González, 2006; Micolta, 2012). Producto de esta situación y ante cualquier medida administrativa de conservación, es necesario disponer de antecedentes biológicos para esta especie, razón que motiva efectuar este trabajo, donde se reportan algunos aspectos como: proporción de sexo, estructura de tallas, relación de talla y peso, estados macroscópicos de madurez gonadal, fecundidad y talla de primera madurez sexual.

\section{MATERIALES Y MÉTODOS}

Se recolectó ejemplares de Pseudocurimata lineopunctata por parte de pescadores artesanales, en los tributarios del río Anchicayá, de forma mensual, entre febrero y octubre 2007. Para la captura se empleó atarraya (con ojo de malla de $1 \mathrm{~cm}$ ), chinchorro (ojo de malla de un cuarto de pulgada), canastos (arte de pesca propio de la costa pacífica construida en fibra vegetal de $50 \mathrm{~cm}$ diámetro y $30 \mathrm{~cm}$ de profundidad) y toldillo (arte de pesca que consta de un paño de $3 \mathrm{~m}$ con ojo de malla $0.3 \mathrm{~mm}$ ); estos artes de pesca pueden llegar a ser excluyentes por el tamaño del ojo de malla de cada arte y el lugar en donde se empleen.

Los organismos fueron trasladados vivos en recipientes plásticos con aireación hasta el laboratorio de Reproducción de Peces Nativos Omar Barona Murillo de la Universidad 
del Pacífico. A cada ejemplar capturado se registró su longitud total $\left(\mathrm{L}_{\mathrm{t}}\right)$ y estándar $\left(\mathrm{L}_{\mathrm{e}}\right)$, con un ictiómetro $( \pm 1 \mathrm{~cm})$ y su peso total $\left(\mathrm{P}_{\mathrm{t}}\right)$ con una balanza (Kern modelo PCB $1000 \pm$ $0.1 \mathrm{~g})$. Mediante disección y observación de las gónadas, se determinó el sexo y se categorizó su estado de madurez sexual macroscópico (EMSM), de acuerdo a los criterios propuestos por Nikolsky (1963). Las gónadas fueron extraídas, pesadas $\left(\mathrm{P}_{\mathrm{g}}\right)$ con balanza analítica Setra modelo BL-410s (0.002 g) y preservadas en formalina al $10 \%$.

Se determinó, la proporción de sexos total, mensual. Mediante una prueba de chi-cuadrado con bondad de ajuste, se estimó el grado de significancia para la relación esperada de 1:1 entre hembras y machos (Zar, 1999). También, se estableció la distribución de longitudes y pesos, así como la proporción de machos y hembras según longitud y peso.

Para hembras y machos se determinó la relación potencial para la $\mathrm{L}_{t} / \mathrm{P}_{t}$, que fue comparada con un análisis de covarianza (ANCOVA; Zar, 1999). Para evaluar la significancia estadística del exponente isométrico (b), éste se analizó con la función propuesta por Pauly (1984):

$$
\mathrm{t}=\frac{\text { s.d. }(\mathrm{x})}{\text { s.d.(y) }} * \frac{\left|\mathrm{b}_{\mathrm{i}}-3\right|}{\sqrt[2]{1-\mathrm{r}^{2}}} * \sqrt[2]{\mathrm{n}_{\mathrm{i}}-2}
$$

Donde t es el estadístico t-student; s.d. (x) e (y) corresponden a la desviación estándar del logaritmo de la $\mathrm{L}_{t}$ y del $\mathrm{P}_{\mathrm{t}}$ para cada grupo analizado; $n_{i}$ es el número de peces muestreados; $b_{i}$ es el valor ajustado de $b \mathrm{y} \mathrm{r}^{2}$ es el ajuste potencial del coeficiente de determinación.

Se determinó el índice gonadosomático (IGS) y gonádico (IG) por las relaciones:

$$
\text { IGS }=\frac{\mathrm{P}_{\mathrm{g}}}{\mathrm{P}_{\mathrm{t}}-\mathrm{P}_{\mathrm{t}}} * 100 \quad \mathrm{IG}=\frac{\mathrm{P}_{\mathrm{g}}}{\mathrm{L}_{\mathrm{t}}^{3}} * 10000
$$

Para el cálculo de la talla de primera madurez sexual, se aplicó el criterio propuesto por Balbontín y Fischer (1981), que considera a los ejemplares sexualmente activos, que en el caso de las hembras, se consideró a las que presentaban estados de madurez vitelogénico, maduro, maduro hidratado y/o folículos post-ovulatorios (diámetros de ovocitos $\geq 1.2 \mathrm{~mm}$ ). Los datos fueron ajustados al siguiente modelo logístico

$$
P_{(1)}=\frac{1}{1+e^{b 0+b \mid * 1}}
$$

La fecundidad total o absoluta corresponde al número de ovocitos susceptibles de ser desovados por un pez en la temporada de puesta (Balbontin \& Fischer, 1981). En las hembras en EMSM V, se extrajo una submuestra de la gónada equivalente a $0.5 \mathrm{~g}$ de su parte central, porción que fue pesada en una balanza analítica de precisión $( \pm 0.001 \mathrm{~g})$. En esta submuestra, los ovocitos se separan de sus lamelas ovocitarias por acción mecánica y fueron contabilizados en una cámara Bogorov, con ayuda de un estereoscopio marca Konus modelo St 30-2L. Se contabilizaron sólo los ovocitos que tenía un diámetro mayor a $1200 \mu \mathrm{m}$, donde se reconoce que el proceso de vitelogénesis ya ha concluido.

Para determinar la fecundidad absoluta (F) se aplicó la expresión (Oliva, Carbajal, \& Tresierra, 1986):

$$
\mathrm{F}=\frac{1}{\mathrm{~N}} \sum_{\mathrm{i}=1}^{\mathrm{n}} \frac{\mathrm{NO}_{\mathrm{i}}}{\mathrm{PSM}_{\mathrm{i}}} * \mathrm{P}_{\mathrm{g}}
$$

Donde $\mathrm{NO}_{\mathrm{i}}$ es el número de ovocitos de la submuestra i y PSM $_{\mathrm{i}}$ es el peso de la submuestra i. Además, se determinó la curva de mejor ajuste entre las relaciones de fecundidad con $\mathrm{P}_{t}$ y con $\mathrm{L}_{t} \mathrm{y}$ se estimó la fecundidad relativa, que se expresó como el número de ovocitos por unidad de peso del pez (Nikolsky, 1963).

\section{RESULTADOS}

Como producto de las campañas realizadas entre febrero y octubre del 2007, se capturó un total de 616 ejemplares de $P$. lineopunctata; los meses en los que hubo una mayor captura fueron agosto y septiembre con 156 y 226 ejemplares, respectivamente (Fig. 1).

Se determinó el sexo en 310 ejemplares. La proporción de hembras (52.6\%) y machos (47.4 \%) expresa una proporción 1:1 ( $\mathrm{P}<0.05$; Fig. 2). En los meses de julio y octubre no hubo 


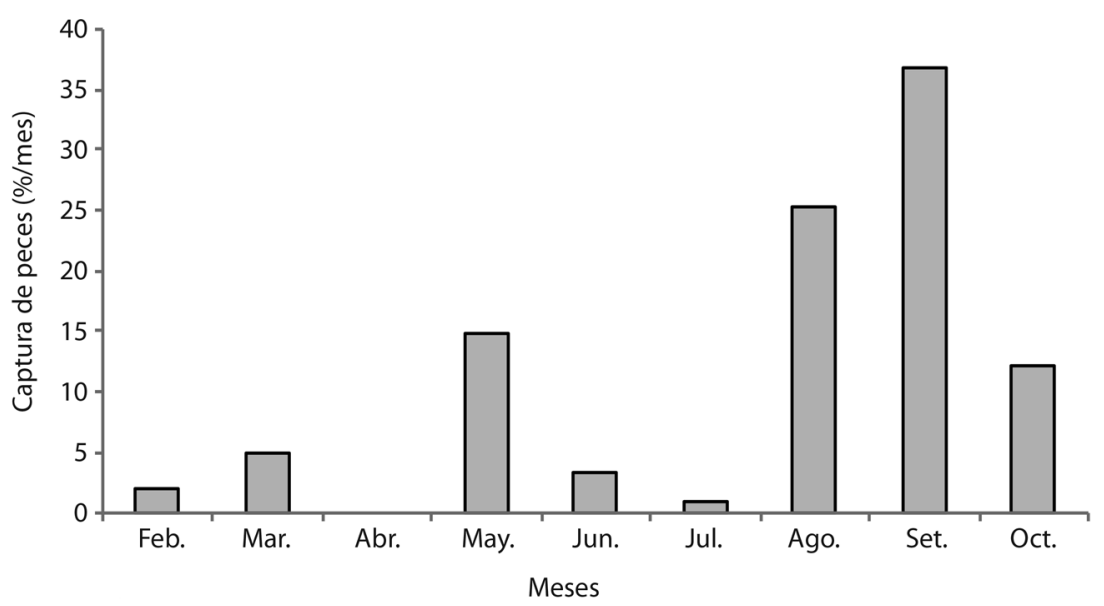

Fig. 1. Captura mensual (\%) del total de ejemplares de Pseudocurimata lineopunctata, en río Anchicayá, Valle del Cauca, Colombia.

Fig. 1. Monthly capture (\%) of specimens of Pseudocurimata lineopunctata in Anchicayá river, Valle del Cauca, Colombia.

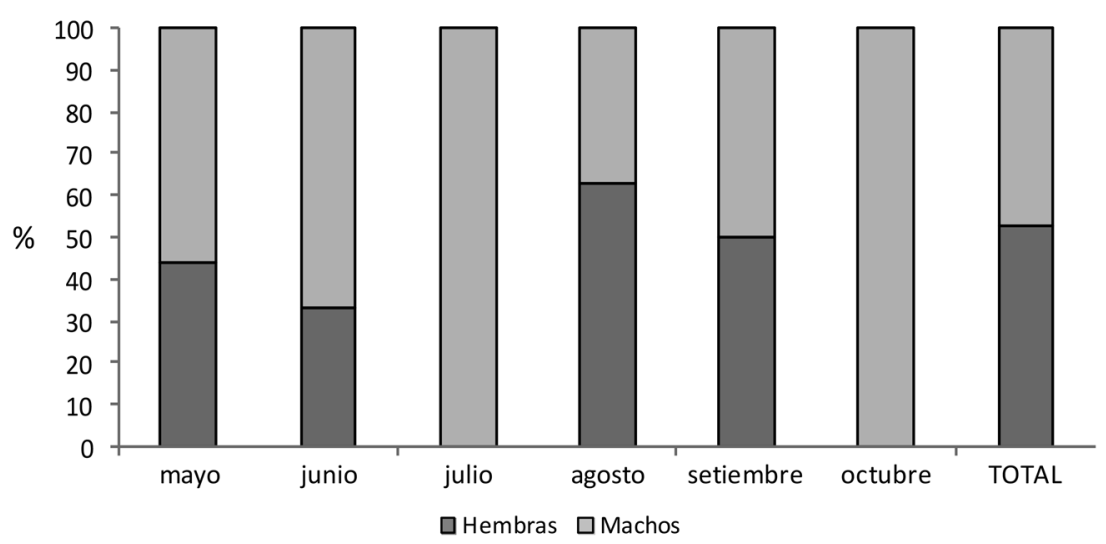

Fig. 2. Porcentaje de machos y hembras de Pseudocurimata lineopunctata, capturados en río Anchicayá, Valle del Cauca, Colombia.

Fig. 2. Percentage of males and females of Pseudocurimata lineopunctata, captured in Anchicayá river, Valle del Cauca, Colombia.

captura de hembras. La proporción sexual en los meses de mayo, junio agosto y septiembre, no fue diferente de la proporción 1:1 $(\mathrm{P}<0.05)$.

Hembras y machos tienen una distinta distribución de tallas $\left(\mathrm{L}_{\mathrm{t}}\right)$, el rango analizado para hembras fue de 11.8 a $18.4 \mathrm{~cm}$, mientras que en machos fue de 10.7 a $16.5 \mathrm{~cm}$ (Fig. 3A). La distribución del $\mathrm{P}_{\mathrm{t}}$ para hembras fue de 25.0 a $67.5 \mathrm{~g}$, y en machos de 16.5 y $55.0 \mathrm{~g}$ (Fig. 3B), mostrando así la existencia de dimorfismo sexual asociado a la talla, las hembras son de un mayor tamaño que los machos.

La proporción de hembras y machos respecto de la talla $\left(\mathrm{L}_{\mathrm{t}}\right)$ y el peso total $\left(\mathrm{P}_{\mathrm{t}}\right)$ es diferencial, lo que muestra un dimorfismo asociado a estas variables. En el caso de la $\mathrm{L}_{\mathrm{t}}$, sólo en el rango de los 13 a los $17 \mathrm{~cm}$ se mantiene la proporción 1:1 de hembras y machos, en las tallas inferiores a los $13 \mathrm{~cm}$ los machos predominan, mientras que en tallas mayores 

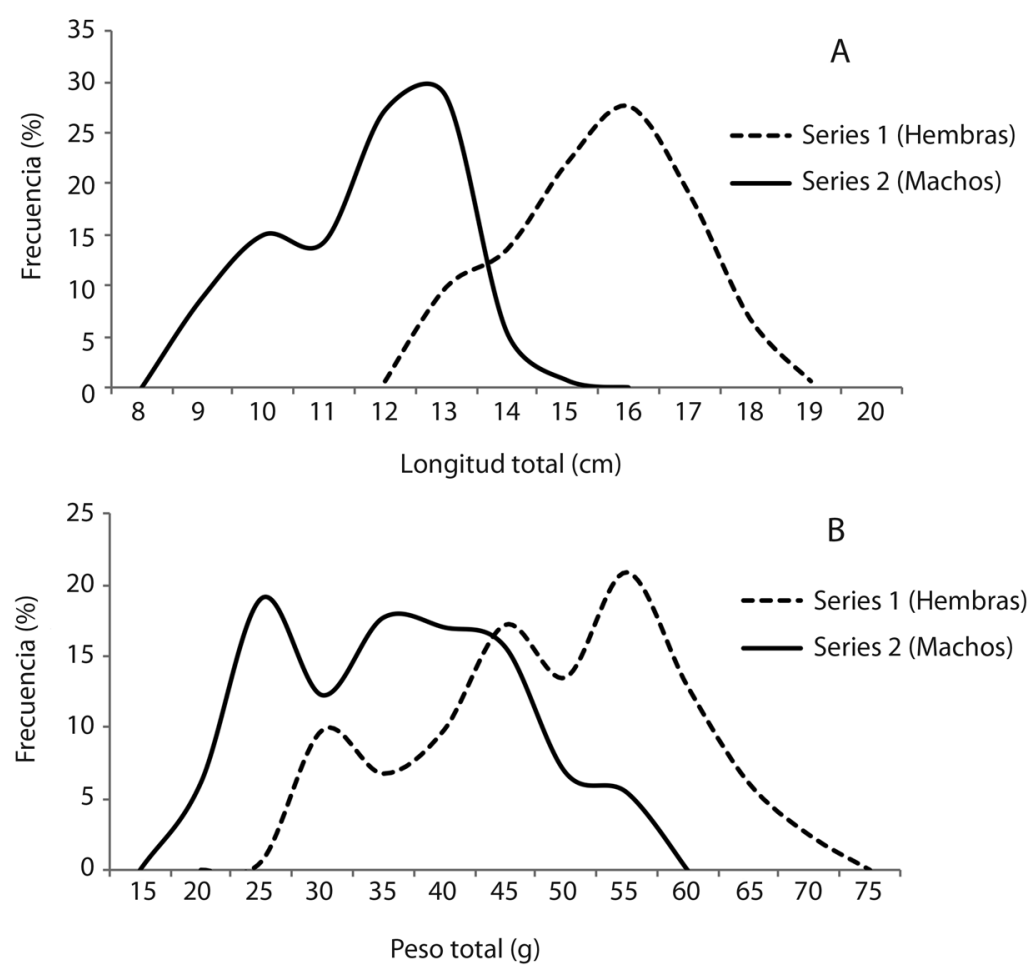

Fig. 3. Distribución de longitud total (A) y peso total (B) en hembras (163) y machos (147) de Pseudocurimata lineopunctata, capturados en río Anchicayá, Valle del Cauca, Colombia.

Fig. 3. Total length distribution (A) and total weight (B) in females (163) and males (147) of Pseudocurimata lineopunctata, captured in Anchicayá river, Valle del Cauca, Colombia.

a los $14 \mathrm{~cm}$, son las hembras las predominantes (Fig. 4A). Al efectuar el mismo análisis respecto del peso total $\left(\mathrm{P}_{t}\right)$, entre los 25 y 45 $\mathrm{g}$ se encontró una proporción 1:1 entre ambos sexos, en los peces menores a los $25 \mathrm{~g}$ es mayor la proporción de machos, mientras que en los ejemplares mayores de $45 \mathrm{~g}$, son las hembras las predominantes (Fig. 4B).

El mejor ajuste para la relación talla-peso para hembras y machos (Fig. 5) está dada por un modelo potencial $\mathrm{P}_{\mathrm{t}}=0.0246 * \mathrm{~L}_{\mathrm{t}}^{2.76}$ $\left(\mathrm{R}^{2}=0.9136\right)$, en hembras la relación es $\mathrm{P}_{\mathrm{t}}=$ $0.059 * \mathrm{~L}_{\mathrm{t}}^{2.4443}\left(\mathrm{R}^{2}=0.8794\right)$, mientras que en machos es $\mathrm{P}_{\mathrm{t}}=0.0179 * \mathrm{~L}_{\mathrm{t}}^{2.8736}\left(\mathrm{R}^{2}=0.9082\right)$. Se determinó la existencia de diferencias significativas entre las pendientes para ambos sexos (Ancova, $\mathrm{Ft}=1.97 ; \mathrm{Fc}=4.13$ ). Las pendientes de la función potencial para hembras y machos, fueron estadísticamente iguales a $3(t=-7.789$ y $t=-1.666$, respectivamente; $\mathrm{P}>0.05)$, lo que determinó un crecimiento isométrico para ambos sexos.

Entre los meses de mayo a octubre se registró para hembras (146) y machos (132) los EMSM (Fig. 6A, Fig. 6B). Un análisis mensual de estos estados, permite visualizar que durante todos los meses muestreados se expresan individuos en estado 5, que corresponde a un estado de madurez avanzada y próxima a la liberación de los gametos, lo que permite inferir que durante todo el año se encuentran individuos de $P$. lineopunctata en desove. Sin embargo, al analizar los índices gonadosómatico, (IGS), gonádico (IG) (Fig. 7) y de condición (K) (Fig. 8 ), se podría postular que habrían dos máximos de desove, uno en los meses de junio-julio y otro en septiembre-octubre.

La captura de individuos no abarcó toda la distribución de tallas, sin embargo, con los datos existentes, se pudo ajustar un modelo 

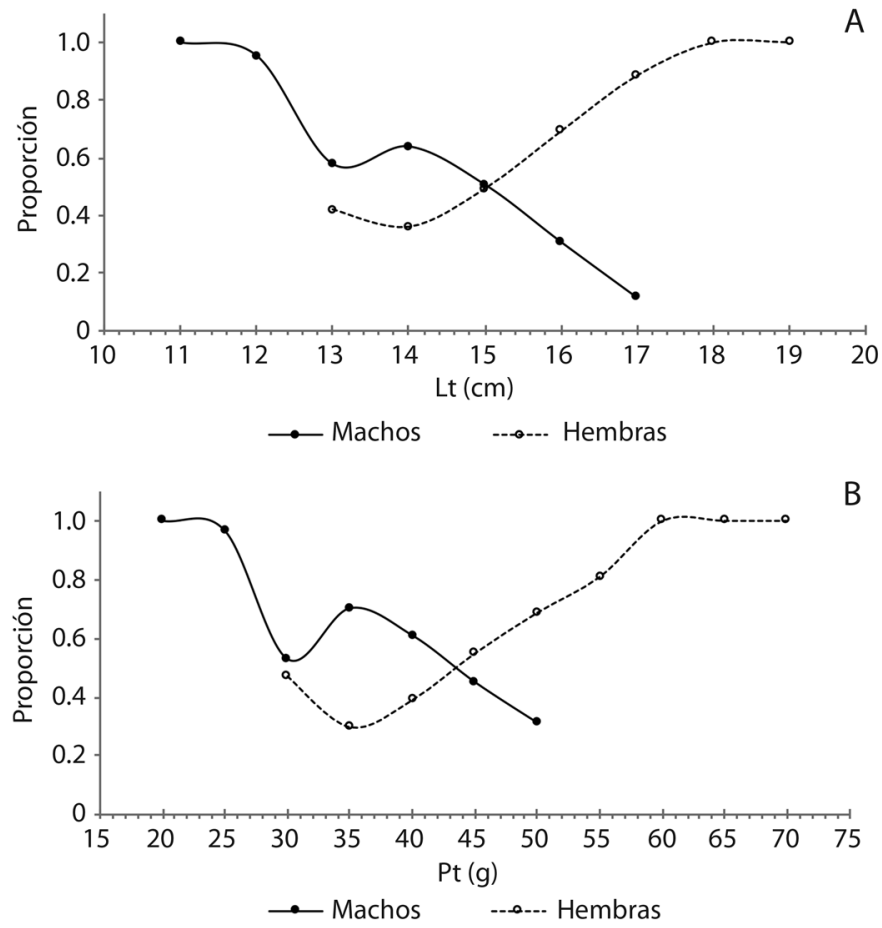

Fig. 4. Proporción de hembras y machos respecto de la longitud total (A) y peso total (B) en Pseudocurimata lineopunctata, capturados en río Anchicayá, Valle del Cauca, Colombia.

Fig. 4. Proportion of males and females with respect to the total length (A) and total weight (B) of Pseudocurimata lineopunctata, captured in Anchicayá river, Valle del Cauca, Colombia.

logístico que permite inferir una TPMS, que se estableció con un total de 163 hembras y 145 machos, correspondiente a los meses de mayo a septiembre. La TPMS para hembra fue determinada en $9.2 \mathrm{~cm}$ y para machos en 10.1 cm de $\mathrm{L}_{\mathrm{t}}$ (Fig. 9).

Para determinar la fecundidad se analizaron 60 hembras, de una Lt promedio de
$14.9 \pm 1.5 \mathrm{~cm}$ y un Pt promedio de $43 \pm 11.3 \mathrm{~g}$. La fecundidad absoluta fue de 3598 ovocitos/ $Q$ y una fecundidad relativa de 84 ovocitos/

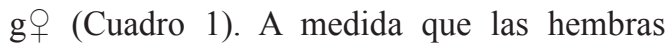
eran de mayor tamaño, producían una mayor cantidad de ovocitos, donde la fecundidad absoluta en función de la Lt y Pt se ajustó a un modelo lineal (Fig. 10A, Fig. 10C), mientras

\section{CUADRO 1}

Longitud total y peso total de hembras utilizadas para determinar la fecundidad en Pseudocurimata lineopunctata, capturadas en río Anchicayá, Valle del Cauca, Colombia

TABLE 1

Total length and total weight of females used to determine fecundity in Pseudocurimata lineopunctata, captured in Anchicayá river, Valle del Cauca, Colombia

\begin{tabular}{lccc}
\multicolumn{1}{c}{ Variable } & Rango & Promedio & Desviación estandar \\
Longitud total $(\mathrm{Lt}, \mathrm{cm})$ & $11.8-18.4$ & 14.9 & 1.55 \\
Peso total $(\mathrm{Pt}, \mathrm{g})$ & $26.0-67.0$ & 43.0 & 11.31 \\
$\mathrm{~N}^{\circ}$ ovocitos / hembra & $846-6851$ & 3598 & 1281 \\
$\mathrm{~N}^{\circ}$ ovocitos / g hembra & $22-163$ & 84 & 32.1 \\
\hline
\end{tabular}



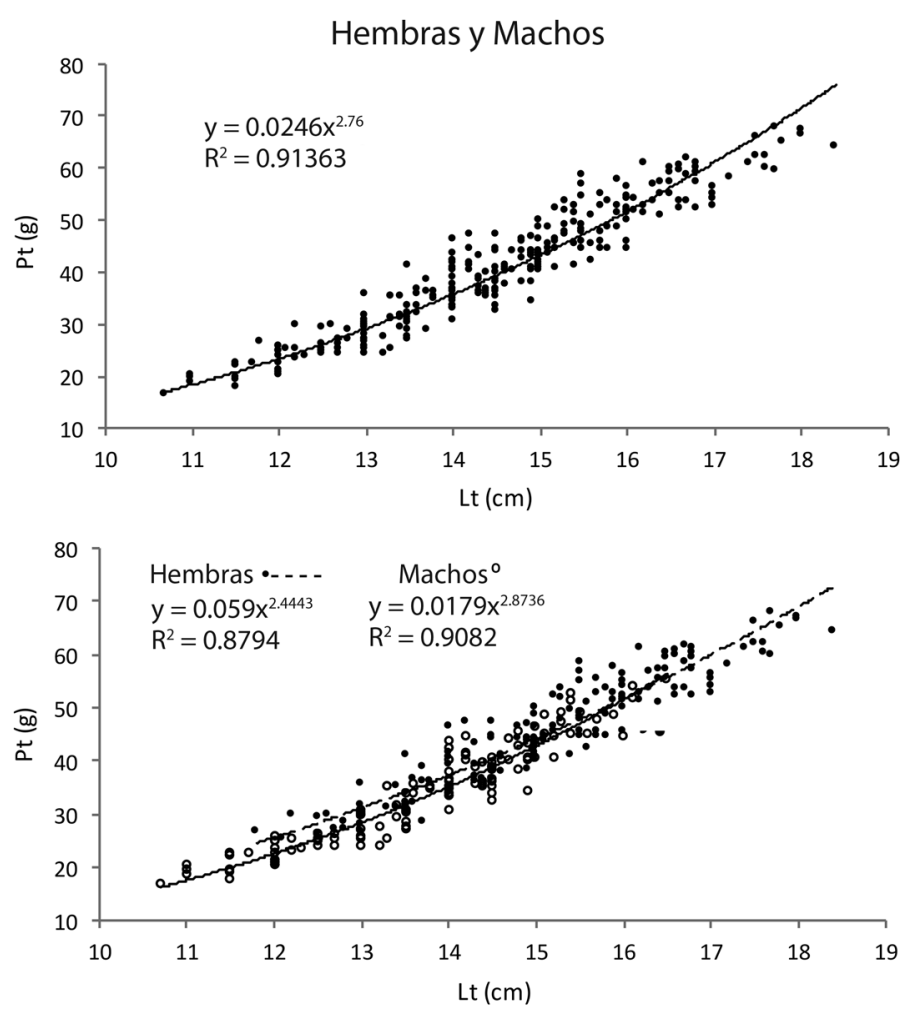

Fig. 5. Relación talla-peso para todos (izquierda) y para hembras y machos (derecha) en Pseudocurimata lineopunctata, capturados en río Anchicayá, Valle del Cauca, Colombia.

Fig. 5. Size-weight relationship for all specimens (left); female and males (right) of Pseudocurimata lineopunctata, captured in Anchicayá River, Valle del Cauca, Colombia.

que la producción de ovocitos/kg de hembra (fecundidad relativa) se ajustó a un modelo logarítmico (Fig. 10B, Fig. 10D).

\section{DISCUSIÓN}

El periodo de lluvias en Buenaventura es mayor entre agosto y diciembre (Lobo-Guerreo, 1993; Climate-data, 2015), situación que incide en que el caudal de los ríos aumenten, que en el caso del río Anchicayá su caudal medio es de $112 \mathrm{~m}^{3} / \mathrm{s}$ (Lobo-Guerrero, 1993). Bajo esta condición ambiental, en estos meses se generan pozos (áreas de inundación) donde los peces ingresan y es más fácil su captura. En algunas ocasiones, cuando llueve en demasía es difícil la captura de peces, debido a que los pozos crecen mucho y genera crecientes repentinas en la cabecera.

El nombre vulgar de $P$. lineopunctata es nayo de pozo, debido a que aprovecha los pozos que se forman en la temporada de lluvias. En estos pozos se encuentran ejemplares de talla mediana y grande (maduros), pero no se han capturado peces pequeños, a pesar que se ha usado malla de $2 \mathrm{~mm}$, situación que motiva ejecutar estudios que permitan dilucidar la ubicación de los juveniles de esta especie.

La proporción entre sexos es generalmente $1: 1$, lo demuestran estudios realizados en peces marinos y dulceacuícolas (Nikolsky, 1963; Flores \& Rojas, 1985; Arellano-Martínez et al., 2001; Rodriguez-Olarte, Taphorn, \& Marrero, 2001); sin embargo, hay reportes en que esta proporción puede cambiar (Pérez \& Parra, 
HEMBRAS (A)
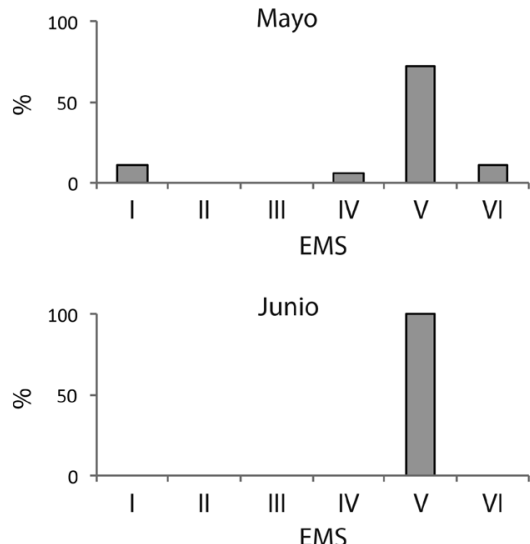

EMS

MACHOS (B)
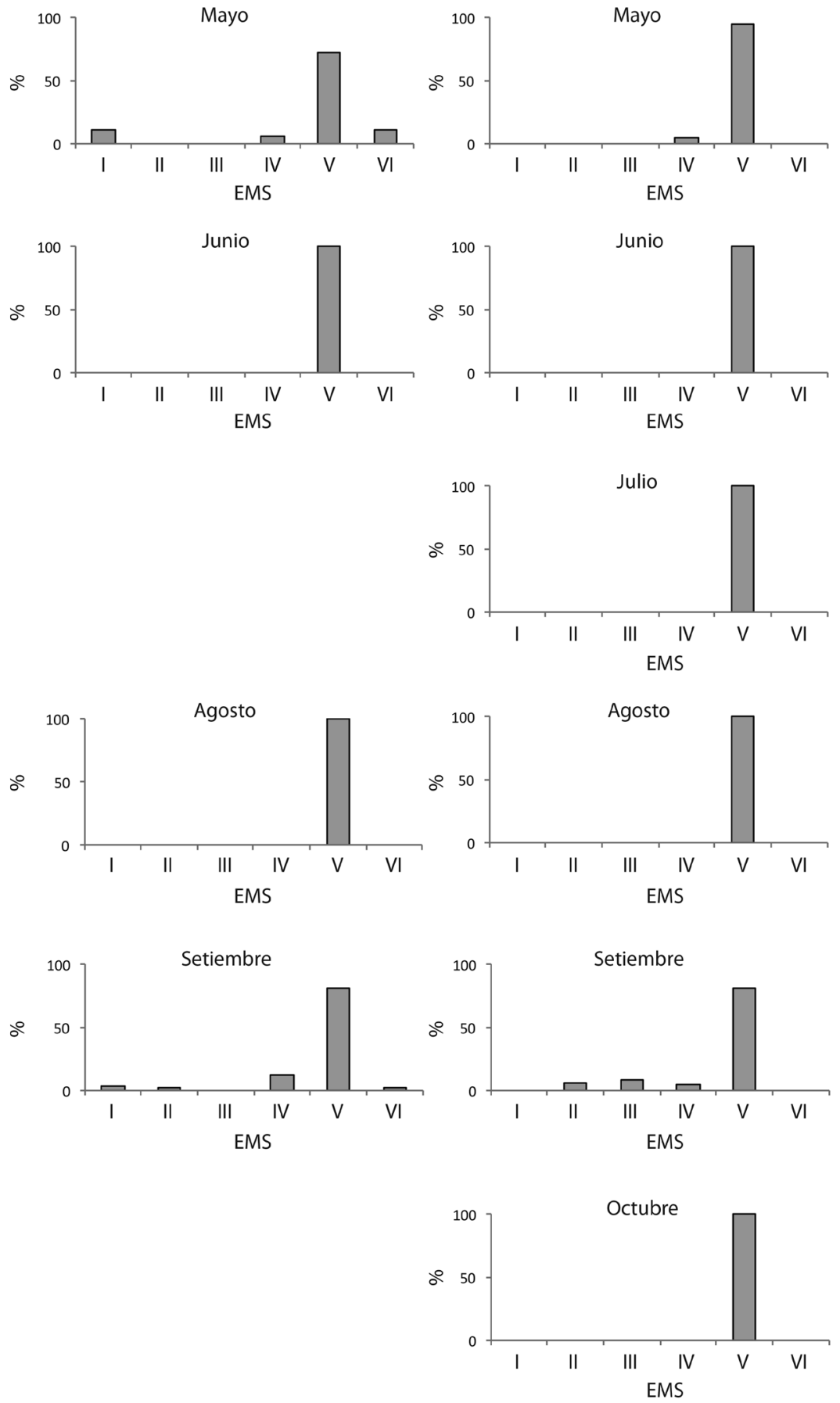

Fig. 6. Estados de madurez sexual macroscópica (EMSM, \%) mensual para hembras (A) y machos (B) de Pseudocurimata lineopunctata, capturados en río Anchicayá, Valle del Cauca, Colombia.

Fig. 6. Monthly macroscopic sexual maturity stages (EMSM, \%) for females (A) and males (B) of Pseudocurimata lineopunctata, captured in Anchicayá river, Valle del Cauca, Colombia. 

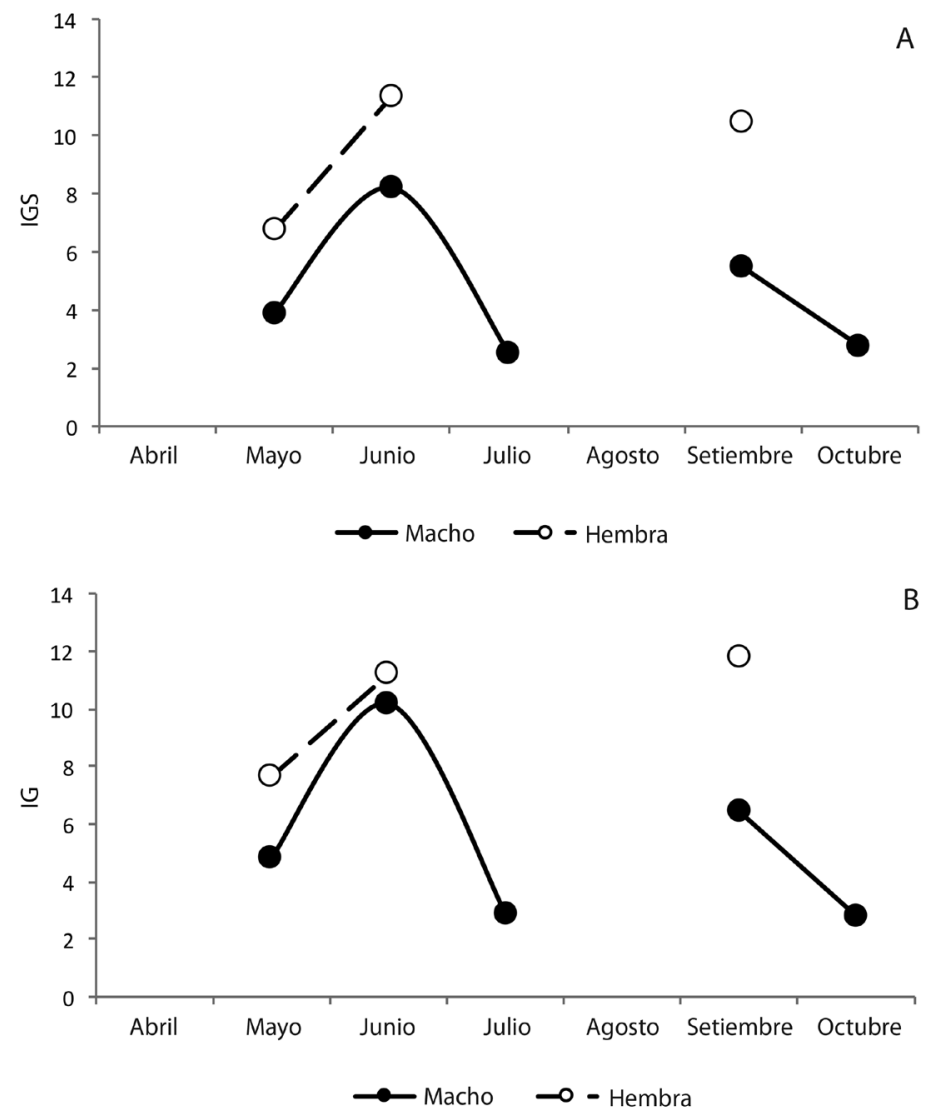

Fig. 7. Índice gonadosomático (IGS, A) e índice gonádico (IG, B) en hembras y machos de Pseudocurimata lineopunctata, capturados en río Anchicayá, Valle del Cauca, Colombia.

Fig. 7. Gonadosomatic index (IGS, A) and gonadic index (IG, B) in female and males of Pseudocurimata lineopunctata, captured in Anchicayá river, Valle del Cauca, Colombia.

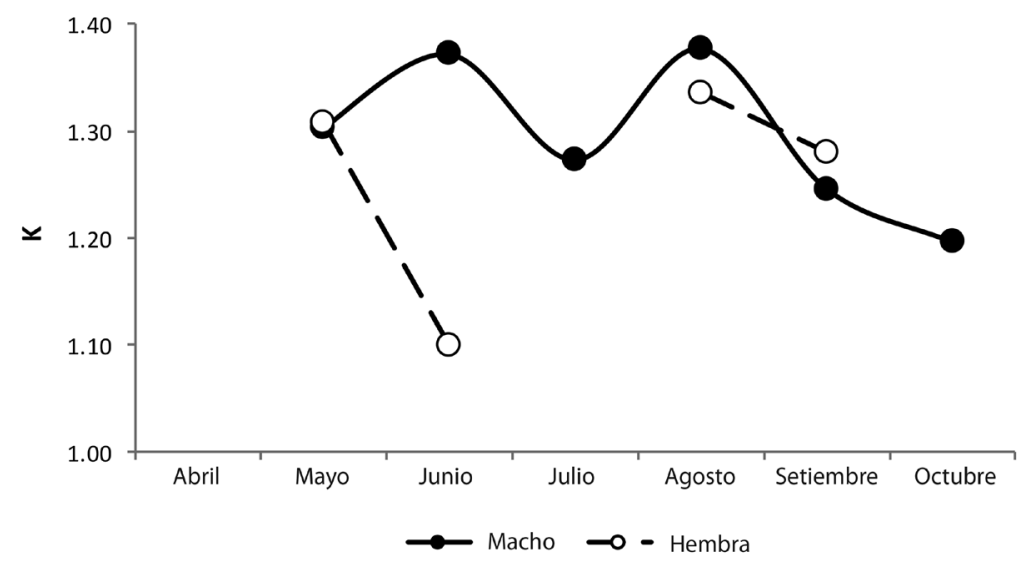

Fig. 8. Índice de condición (K) en hembras y machos de Pseudocurimata lineopunctata, capturados en río Anchicayá, Valle del Cauca, Colombia.

Fig. 8. Condition index (K) in female and males of Pseudocurimata lineopunctata, captured in Anchicayá river, Valle del Cauca, Colombia. 

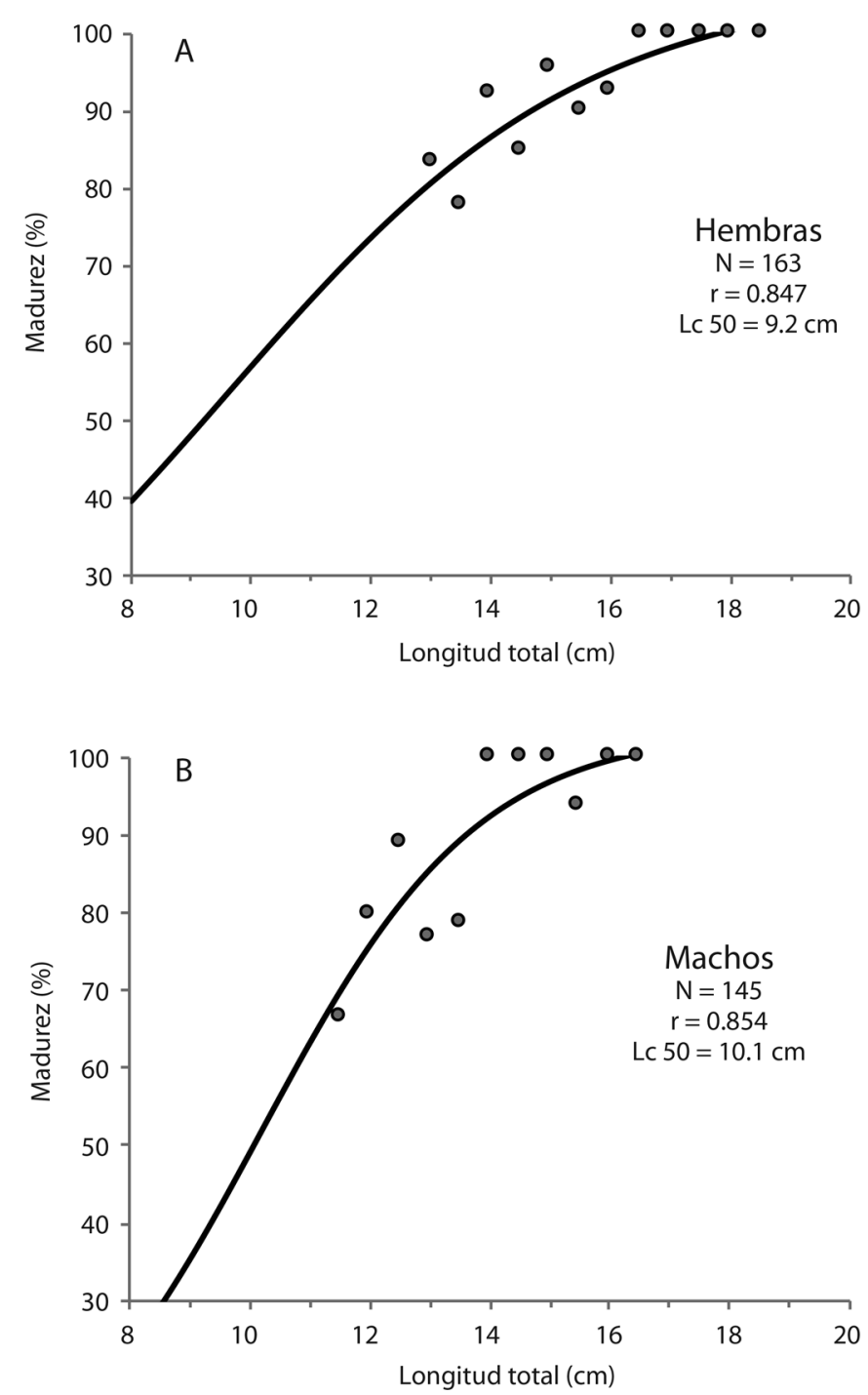

Fig. 9. Talla de primera madurez sexual en hembras (A) y machos (B) de Pseudocurimata lineopunctata, capturados en río Anchicayá, Valle del Cauca, Colombia, determinada por el método del $50 \%$ de ejemplares maduros.

Fig. 9. Size of first sexual maturity in females (A) and males (B) of Pseudocurimata lineopunctata, captured in Anchicayá river, Valle del Cauca, Colombia, by the method of $50 \%$ of mature specimens.

2002; Trujillo-Jiménez \& Toledo, 2007; Casimiro, García, Almeida, \& Orsi, 2011, Ferriz, Bentos, Fernández, \& López, 2011; Rodriguez et al., 2011), lo que dependería de una población a otra o también variar en determinados momentos durante el año.

En todos los meses en que se capturó y determinó hembras y machos, no hubo diferencia en la proporción de sexo, excepto en los meses de julio y octubre, en que no hubo captura de hembras. El dimorfismo sexual asociado a la talla en esta especie se presenta de igual manera que en $P$. boulengeri (Chicaiza \& Flores, 2016) y en Curimatella lepidura (de Alvarenga, Bazzoli, Santos, \& Rizzo, 2006). Los ejemplares de $P$. lineopunctata son de un menor tamaño que $P$. boulengeri (Chicaiza \& Flores, 2016).En hembras y machos, se manifiesta 

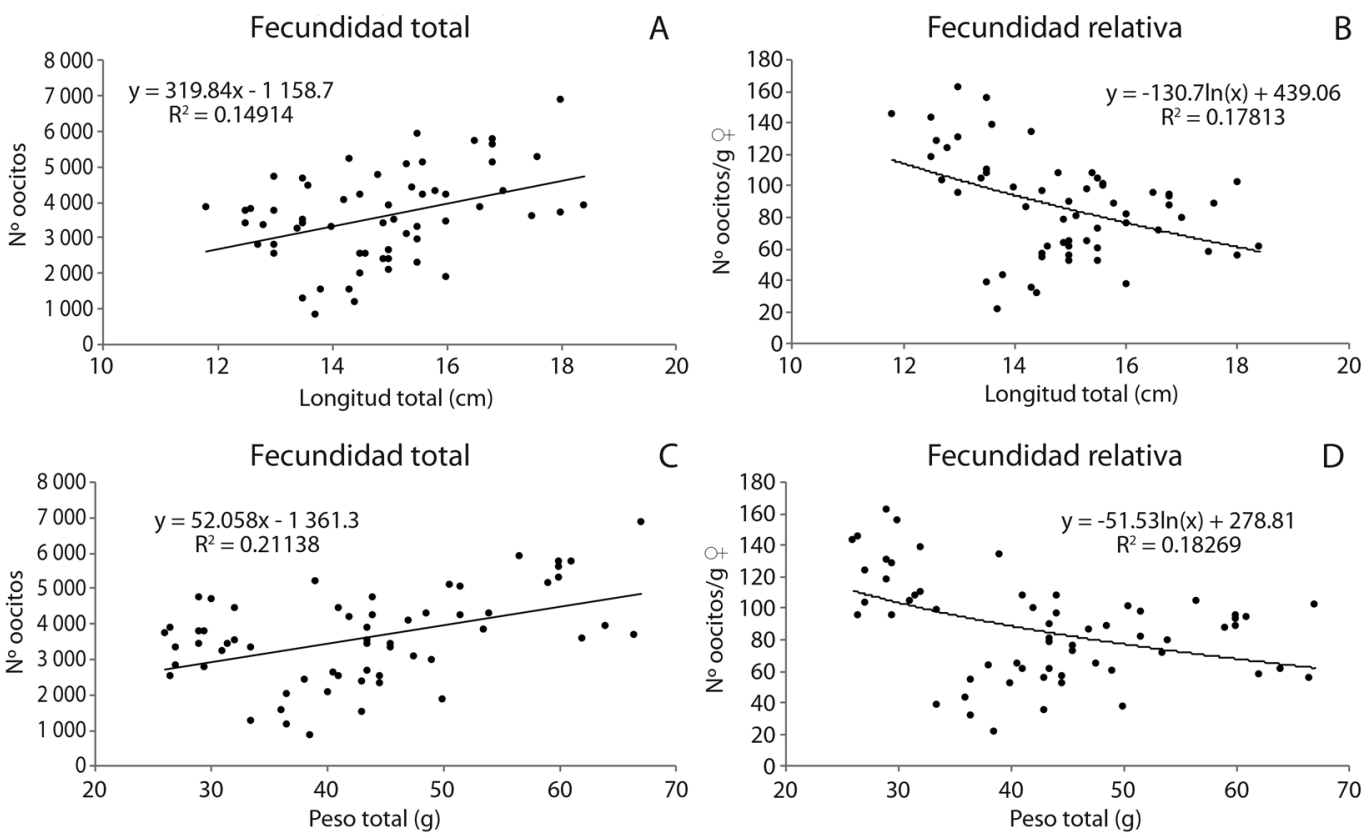

Fig. 10. Fecundidad absoluta y relativa en función de la longitud total (A, C) y peso total (B, D) en Pseudocurimata lineopunctata, capturados en río Anchicayá, Valle del Cauca.

Fig. 10. Absolute and relative fecundity depending on the total length (A, C) and total weight (B, D) in Pseudocurimata lineopunctata, captured in Anchicayá river, Valle del Cauca, Colombia.

un crecimiento isométrico, semejante a lo que ocurre en $P$. boulengeri (Chicaiza \& Flores, 2016) y en Psectrogaster ciliata (RodríguezOlarte et al., 2001).

En ambientes tropicales, la mayoría de las especies que desovan en ambiente lótico tienen migración reproductiva, con un periodo de desove corto y total, situación que contrasta con lo que ocurre en peces que desovan en un ambiente léntico, que no migran, la estación de desove es media y extendida, con desoves múltiples (Godinho, Lamas, \& Godinho, 2010). Las especies que migran, por lo general esta conducta se asocia con las fluctuaciones del nivel medio del río (Menezes \& Vazzoler, 1992) y desovan una vez al año, actividad que generalmente se correlaciona con los periodos de aguas altas en los ríos, mientras que las no migratorias, pueden desovar durante todo el año (Blanco-Parra \& Bejarano-Rodríguez, 2006). En P. ciliata, C. lepidura y P. boulengeri (Curimatidae), la madurez gonadal se ha asociado temporalmente con el periodo de lluvias (Rodríguez-Olarte et al., 2001; de Alvarenga et al., 2006; Chicaiza \& Flores, 2016).

Steindachnerina insculpta tiene un período prolongado de actividad reproductiva que se extiende de septiembre a marzo, con un desove fraccionado (Ribeiro, Santos, \& Bazzoli, 2007), en P. boulengeri (Chicaiza \& Flores, 2016) el desove ocurre de octubre a febreromarzo, tal como en la mayoría de las especies de Curimatidae. En P. lineopunctata los antecedentes disponibles no son consistentes para determinar una época de desove con precisión.

La talla de primera madurez sexual en $P$. lineopunctata es menor que la registrada para P. boulengeri (Chicaiza \& Flores, 2016). Es importante destacar que no se capturó ejemplares bajo la talla de primera madurez reproductiva determinada por el modelo logístico, lo que podría inferir que el arte de pesca discriminó significativamente las tallas pequeñas, sin embargo, se utilizó en algunas situaciones 
malla de $2 \mathrm{~mm}$ (mosquitera). De acuerdo a estos antecedentes, se hace necesario profundizar sobre el reclutamiento de las diferentes tallas y su distribución en el cuerpo de agua.

Los valores de fecundidad total y relativa en $P$. lineopunctata es bastante menor respecto de otras especies de Curimatidae. En Curimatella lepidura, el valor medio de fecundidad total fue de $60994.9 \pm 27$, con 142.7 ovocitos/ hembra, y una fecundidad relativa de 1294.1 \pm 437.5 por $\mathrm{g} /$ hembra (Andrade et al., 2010), mientras que en Cyphocharax voga, la fecundidad total fue de 74920 ovocitos/hembra (Hartz, Martins, \& Peret, 1994).

El río Anchicayá tiene diferentes intervenciones, una de las más importantes es el efecto que produce la compañía Celsia S.A., que genera, transmite, distribuye y vende electricidad en Colombia y Panamá, a través de tres termoeléctricas y 23 centrales hidroeléctricas, dos de éstas últimas, se ubican en el Alto y Bajo Anchicayá. Estas centrales se ubican en un hábitat de selva húmeda tropical, correspondiente al tercer lugar en pluviosidad en el mundo. La Central Hidroeléctrica Bajo Anchicayá, ubicada dentro del perímetro del Parque Natural Los Farallones, consciente del impacto en la fauna acuática y en especial en la íctica, que producen estas obras hidráulicas, apoya un programa orientado a la reproducción en cautiverio de las principales especies ícticas nativas registradas en el río Anchicayá, de modo de obtener juveniles que en el futuro permitan un repoblamiento (EPSA, 2007). Estas acciones son un avance, pero se requiere de mayor investigación y descripción de los aspectos biológicos y reproductivos de las especies ícticas que habitan esta hoya hidrográfica.

\section{AGRADECIMIENTOS}

Los autores agradecen a la Universidad del Pacífico por su apoyo logístico y a EPSA Empresa de Energía del Pacífico por la dotación del Laboratorio de Reproducción de Peces Nativos Omar Barona Murillo de acuerdo al contrato EP 289 de 2006.

\section{RESUMEN}

Pseudocurimata lineopunctata representa un importante aporte nutricional para las comunidades locales en Colombia y Ecuador. A pesar que su captura anual es baja (590 kg/año), no hay restricciones sobre el tamaño mínimo, y esta especie está catalogada como vulnerable en riesgo de extinción moderada por la Corporación Autónoma Regional del Valle del Cauca (Colombia). Para apoyar los programas de conservación, se requiere de datos biológicos de las especie. Por lo tanto, los objetivos de este estudio fueron describir la proporción de sexos, la distribución, el tamaño en relación al peso, las fases macroscópicas de la madurez reproductiva, fecundidad y talla de primera madurez sexual para $P$. lineopunctata. Las muestras de peces fueron capturados con redes por nueve meses (Febrero-Octubre) en 2007. La longitud total (Lt) de los peces capturados varió entre 10.7 y $16.5 \mathrm{~cm}$, con peso total $(\mathrm{Pt})$ entre 25.0 y $67.5 \mathrm{~g}$. Las hembras representaron el $52.6 \%$ del grupo de muestra mientras que los machos el $47.4 \%$, y se observaron diferencias en la proporción de sexos en relación con el tamaño del pez. Durante todos los meses muestreados se capturaron ejemplares en estado de madurez avanzado. Con base en el análisis de los índices gonadosómatico, (IGS), gonádico (IG) y de condición (K), se postula que habría dos máximos de desove, uno de junio-julio y otro en septiembre-octubre. La talla de primera madurez sexual para las hembras fue determinada en $9.2 \mathrm{~cm}$ y para los machos en $10.1 \mathrm{~cm}$ de Lt. La fecundidad absoluta fue estimada en 3598 ovocitos/ + , con una fecundidad relativa de 84 ovocitos $/ g$ ㅇ. Se hace necesario realizar investigaciones adicionales que aumenten la información sobre la reproducción de esta especie, con la finalidad de apoyar futuros programas de repoblamiento.

Palabras clave: proporción sexos, relación talla-peso, reproducción, desove, talla primera madurez, fecundidad.

\section{REFERENCIAS}

Andrade, D. R., Junior, V., Godinho, H. P., Tonini, W. C. T., \& Burket, D. (2010). Fecundity of manjuba Curimatella lepidura (CURIMATIDAE) in the Três Marias reservoir, São Francisco River, MG. Arquivo Brasileiro de Medicina Veterinária e Zootecnia, 62(6), 1401-1408. https://dx.doi.org/10.1590/ S0102-09352010000600016

Angulo, J. A., \& García, L. N. (2010). Inventario de la ictiofauna de la cuenca media del río Anchicayá y selección de especies con potencial para cultivo. Revista Bioetnia, 7(2), 104-110.

de Alvarenga, É. R., Bazzoli, N., Santos, G. B., \& Rizzo, E. (2006). Biologia reprodutiva e alimentação de Curimatella lepidura (Eigenmann \& Eigenmann) (Pisces, Curimatidae) no reservatório de Juramento, Minas Gerais. Revista Brasileira 
Zoologia, 23, 314-322. https://dx.doi.org/10.1590/ S0101-81752006000200002

Arellano-Martinez, A., Rojas-Herrera, A., García-Dominguez, F., Ceballos-Vasques, B., \& Villalejo-Fuerte, M. (2001). Ciclo reproductivo del pargo lunarejo Lutjanus guttatus (Steindachner, 1869) en las costas de Guerrero, México. Revista de Biología Marina \& Oceanografia, 36(1), 1-8. https://dx.doi.org/10.4067/ S0718-19572001000100001

Autoridad Nacional de Acuicultura y Pesca (AUNAP). (2013). Tallas mínimas de captura y recomendaciones técnicas para el aprovechamiento sostenible de los recursos pesqueros de Colombia. Recuperado de http://sepec.aunap.gov.co/Archivos/Cartilla\%20\%20 TALLAS\%20MINIMAS DIGITAL\%20-\%20REFERENCIADA \%20V3\%20(2).pdf

Autoridad Nacional de Acuicultura y Pesca (AUNAP). (2014). Servicio Estadístico Pesquero Colombiano SEPEC. Boletín Estadístico, enero-junio 2014. Recuperado de http://sepec.unimagdalena.edu.co/Archivos/Boletin\%20Enero\%20-\%20Junio\%202014.pdf

Blanco-Parra, M. P., \& Bejarano-Rodríguez, I. (2006). Alimentación y reproducción de las principales especies ícticas del río Mesay durante el período de "aguas altas". Revista Biología Tropical, 54(3), 853-859.

Balbontín, F., \& Fisher, W. (1981). Ciclo sexual y fecundidad de la merluza, Merluccius gayi gayi, en la costa de Chile. Revista Biología Marina, 17(3), 285-334.

Casimiro, A. C. R., García, D. A. Z., Almeida, F. S., \& Orsi, M. L. (2011). Reproductive aspects of Moenkhausia intermedia Eigenmann, 1908 (Pisces, Characidae) in the Upper Paraná River Basin, Brazil. Zoology, 1-8.

Castillo, L. S., \& González, A. M. 2007. Avances en la implementación del Plan de Acción en Biodiversidad del Valle del Cauca. Agenda de Investigación en Biodiversidad y vertebrados amenazados. Cali, Colombia: CVC Dirección Técnica Ambiental.

CAMAWA Asociación de Autoridades Wounaan del Pacífico y OREWA Organización Regional Embera Wounaan. (2000). Informe Final del Proyecto del Plan de Vida de las Comunidades Indigenas del Bajo San Juan. Recuperado de http://observatorioetnicocecoin. org.co/files/CAMAWA \%20-\%20Plan $\% 20 \mathrm{de} \% 20$ Vida.pdf

Chaves, M. E., \& Santamaría, M. (Ed) (2006). Informe Nacional sobre el Avance en el Conocimiento y la Información de la Biodiversidad 1998-2004. 2 Tomos. Bogotá D.C., Colombia: Instituto de Investigación en Recursos Biológicos Alexander von Humboldt.

Chicaiza, D., \& Flores, H. (2016). Parámetros biológicos de Pseudocurimata boulengeri (Characiformes, Curimatidae) en el embalse Chongón, Ecuador. Revista de Biología Tropical, 64(1), 131-146.
Climate-Data. Org. (2015). Datos Climáticos Mundiales. Recuperado de http://es.climate-data.org/region/69/

Critical Ecosystem Partnership Fund. (2007). Análisis de cinco años de inversiones del CEPF en la ecorregión Tumbes-Chocó -Magdalena. Corredor de Conservación Chocó-Manabi Colombia y Ecuador. Recuperado de http://www.cepf.net/Documents/ final_chocomanabi_assessment_march07_sp.pdf

Corporación Autónoma Regional del Valle del Cauca (C.V.C.) y la Universidad del Valle. (1998). Plan integral de ordenamiento y manejo sostenible con participación comunitaria de la cuenca hidrográfica del Río Anchicayá (Convenio Nº86/96). Colombia: Departamento del Valle del Cauca, Municipios de Buenaventura y Dagua.

Empresa de Energía del Pacífico. EPSA. (2007). Informe de Sostenibilidad. Recuperado de https://www.unglobalcompact.org/system/attachments/790/original/ COP.pdf?1262614221

Ferriz, R. A., Bentos, C. A., Fernández, E. M., \& López, G. R. (2011). Reproducción y dinámica poblacional de Cheirodon interruptus (Ostariophysi: Characidae) en el arroyo El Portugués, alta cuenca del río Samborombón, Argentina. Latin American Journal of Aquatic Research, 39(1), 151-160.

Flores, H. \& Rojas, P. (1985). Talla de primera madurez, época de desove y fecundidad del bacalao de Juan Fernández Polyprion oxygeneios (Bloch y Schneider, 1801) (Pisces: Percichthyidae). In P. Arana (Ed.), Investigaciones Marinas en el Archipiélago de Juan Fernández, Escuela de Ciencias del Mar (pp. 311322). Valparaíso: Universidad Católica de Valparaíso.

Froese, R. \& Pauly, D. (Ed) (2015). FishBase. World Wide Web electronic publication. Recuperado de www. fishbase.org, version (04/2015).

Godinho, A. L., Lamas, I. R., \& Godinho, H. P. (2010). Reproductive ecology of Brazilian freshwater fishes. Environmental Biology of Fishes, 87(2), 143-162.

González. (2006). Vertebrados Amenazados. Peces dulceacuícolas amenazados del Valle del Cauca, categorizados por especialistas del departamento. Recuperado de http://www.cvc.gov.co/index.php/ servicio-al-ciudadano/inscriba-departamento-degestion-ambiental/175-biodiversidad/especies/fauna/ fauna-amenazada/1943-vertebrados-amenazados

Grueso, H., \& Lamouroux, S. L. (2008). Adaptación al cautiverio del nayo de pozo (Pseudocurimata lineopuctata) con fines reproductivos. Revista Colombiana Ciencias Pecuarias, 21, 508.

Hartz, S. M., Martins, A. G., \& Peret, A. C. (1994). Fecundidade de Cyphocharax voga (Hensel, 1869) na lagoa Emboaba, Rio Grande do Sul, Brasil (Characiformes, Curimatidae). Iheringia, Série Zoologia, 76, 161-165. 
Instituto de Investigación de Recursos Biológicos Alexander von Humboldt. (2012). Informe sobre el estado de los recursos naturales renovables y del ambiente, componente de biodiversidad, 2010-2011. Recuperado de http://www.humboldt.org.co/images/documentos/ pdf/documentos/iern-biodiversidad-2010-2011.pdf

Lasso, C. A., Rial, A., Matallana, C., Ramírez, W., Señaris, J., Díaz Pulido, A., Corzo, G., \& Machado-Allison, A. (Eds.) (2011). Biodiversidad de la cuenca del Orinoco. II Áreas prioritarias para la conservación y uso sostenible. Bogotá, D.C., Colombia: Instituto de Investigación de Recursos Biológicos Alexander von Humboldt, Ministerio del Ambiente, Vivienda y Desarrollo Territorial, WWF Colombia, Fundación Omacha, Fundación La Salle de Ciencias Naturales e Instituto de Estudios de la Orinoquia (Universidad Nacional de Colombia).

Lobo-Guerreo, A. (1993). Hidrología e Hidrogeología de la Región Pacífica Colombiana. In P. Leyva (Ed), Colombia - Pacífico (Tomo I, pp. 122-134). Colombia: Fondo para la Protección del Medio Ambiente “José Celestino Mutis”, FEN Bogotá, Colombia.

Maldonado-Ocampo, J., Vari, R. P. R., \& Usma, J. S. (2008). Checklist of the Freshwater Fishes of Colombia. Biota Colombiana, 9(2), 143-237.

Maldonado-Ocampo, J. A., Usma, J. S., Villa-Navarro, F. A., Ortega-Lara, A., Prada-Pedreros, S., Jiménez, L. F., Jaramillo-Villa, U., Arango, A., Rivas, T., \& Sánchez Garcés, G. C. (2012) Peces Dulceacuícolas del Choco Biogeográfico de Colombia. Bogotá D.C., Colombia: WWF Colombia, Instituto de Investigación de Recursos Biológicos Alexander von Humboldt (IAvH), Universidad del Tolima, Autoridad de Acuicultura y Pesca (AUNAP), Pontificia Universidad Javeriana.

Menezes, N. A., \& Vazzoler, A. E. A. D. M. (1992). Reproductive characteristics of Characiformes. In W. Hamlett (Ed.), Reproductive Biology of South American Vertebrates (pp 60-70). New York: Springer.

Ministerio Del Ambiente. (2011). Evaluación Ambiental de los Cantones Eloy Alfaro y San Lorenzo (Cuenca del Rio Santiago y Bogotá). Recuperado de http://www. agua.gob.ec/wpcontent/uploads/downloads/2012/07/ InformeInterMAESenagua.pdf

Micolta, T. (comp) (2012). Caracterización Física, Biológica, Socioeconómica y Cultural de la Cuenca Alta del Río Dagua (Tomo III, Componente Biótico). Consejo Comunitario del Alto Medio Dagua. Recuperado de file://C:/Users/asus/Downloads/bioticovr2.2c-1.pdf

Mojica, J. I., Usma, J. S., Álvarez-León, R., \& Lasso, C. A. (Ed) (2012). Libro rojo de peces dulceacuícolas de Colombia 2012. Bogotá, Colombia: Instituto de Investigación de Recursos Biológicos Alexander von
Humboldt, Instituto de Ciencias Naturales de la Universidad Nacional de Colombia, WWF Colombia y Universidad de Manizales.

Nikolsky, G. V. (1963). The ecology of fishes. New York: Academic Press

Oliva, J. N., Carbajal, J. W., \& Tresierra, A. (1986). Reproducción e histología de gónadas de peces. Trujillo, Perú: Universidad Nacional de Trujillo, Depto. Ciencias Biológicas, Sección de Recursos Acuáticos.

Pauly, D. (1984). Fish population dynamics in tropical waters: a manual for the use with programmable calculators. ICLARM Studies and Reviews, 8, 325.

Perez, G. \& Parra, D. L. (2002). Aspectos Reproductivos de Halichoeres bivittatus (Bloch, 1971) (Pises: Labridae) en Tocuchare, Golfo de Cariaco. Saber, 14(1), 105-112.

Programa Colombia Forestal PCF. (2006). Plan de Manejo Forestal para un área de 23.651 ha de Propiedad del Consejo Comunitario del Alto Guapi en el Municipio de Guapi Departamento del Cauca. Recuperado de http://pdf.usaid.gov/pdf_docs/PNADK927.pdf

Ribeiro, V., Santos, G. B., \& Bazzoli, N. (2007). Reproductive biology of Steindachnerina insculpta (Fernandez-Yépez) (Teleostei, Curimatidae) in Furnas reservoir, Minas Gerais, Brazil. Revista Brasilera de Zoología. 24(1), 71-76.

Rodriguez-Olarte, D., Taphorn, D., \& Marrero, C. (2001). Aspectos de la distribución y ecología reproductiva de Psectrogaster ciliata (Pisces: Curimatidae) en la Orinoquia Venezolana. Bioagro, 13(2), 85-89.

Rodrigues, A., Zoccal, D., Simões, F., \& Orsi, M. (2011). Reproductive aspects of Moenkhausia intermedia Eigenmann, 1908 (Pisces, Characidae) in the Upper Paraná River Basin, Brazil. ISRN Zoology, 1-8. doi: $10.5402 / 2011 / 802794$

Sánchez-Garcés, G., \& Ortega-Lara, A. (2011). Pseudocurimata lineopunctata. In C. A. Lasso, E. Agudelo, L. F. Jiménez-Segura, H. Ramírez-Gil, M. MoralesBetancourt, R. E. Ajiaco-Martínez, F. de Paula Gutiérrez, J. S. Usma, S. E. Muñoz, \& A. I. Sanabria (Eds.) I. Catálogo de los recursos pesqueros continentales de Colombia. Serie Editorial Recursos Hidrobiológicos y Pesqueros Continentales de Colombia (pp. 278-279). Bogotá, D. C., Colombia: Instituto de Investigación de los Recursos Biológicos Alexander von Humboldt (IAvH).

Trujillo-Jiménez, P., \& Toledo, H. (2007). Alimentación de los peces dulceacuícolas tropicales Heterandria bimaculata y Poecilia sphenops (Cyprinidontiformes: Poeciliidae). Revista de Biología Tropical, 55(2), 603-615. 
Tovar, J. A. (2004). Estudio hidrobiológico comparativo (monitoreo), para observar el estado actual del rió Anchicayá, tanto aguas arriba como aguas abajo de la represa del bajo Anchicayá. Colombia: Empresa de Energía del Pacífico ESP. S.A.

Valderrama, N., García, N., Baptiste, E. M. P., Renjifo, L. M., Sánchez Duarte, P., Cárdenas-Toro, J., .. \& \& Toro, J. L. (2014). Especies amenazadas de flora y fauna. In
J. C. Bello, M. Báez, M. F. Gómez, O. Orrego, \& L. Nägele (Eds.), Biodiversidad 2014. Estado y tendencias de la biodiversidad continental en Colombia (pp. 23-24). Bogotá D.C.: Colombia. Instituto Alexander von Humboldt.

Zar, J. H. (1999). Biostatistical Analysis. Fourth edition. Englewood Cliffs, New Jersey: Prentice Hall. 
